\title{
AN ALGEBRA ASSOCIATED TO A COMBINATORIAL GEOMETRY
}

\author{
BY WILLIAM GRAVES ${ }^{1}$
}

Communicated by Gian-Carlo Rota, March 17, 1971

1. Preliminaries. A functor from a category of combinatorial geometries, or equivalently a category of geometric lattices, to a category of commutative algebras will be described, and some properties of this functor will be investigated. In particular, a cohomology will be associated to each point of a geometry and will be derived from the associated algebra.

If $(G, S)$ is a geometry on a set $S[1$, p. 2.4], then $L(G)$, or simply $L$ when no opportunity for confusion exists, denotes the associated geometric lattice of closed subsets of $S$. The rank function of $L$ or $G$ is denoted $r$.

A morphism

$$
\sigma:(G, S) \rightarrow\left(G^{\prime}, S^{\prime}\right)
$$

of geometries is a function

$$
\sigma: S \cup\{0\} \rightarrow S^{\prime} \cup\{0\}
$$

such that $\sigma(0)=0$ and the inverse image of a closed subset of $S^{\prime}$ is a closed subset of $S$. It is precisely the latter condition which is necessary and sufficient $[1$, p. 9.17] to extend $\sigma$ to a strong map $[1$, p. 9.3]

$$
\sigma: L(G) \rightarrow L\left(G^{\prime}\right)
$$

The category of geometries with morphisms defined as above is equivalent to the category of geometric lattices and strong maps. These two categories will be used interchangeably throughout.

2. The functor $(G, S) \rightarrow A(G)$. Let $k$ be a commutative ring (with 1$)$. For any geometry $(G, S)$, let $P(G)$ be the symmetric $k$-algebra on the free $k$-module on $S$; that is, the $k$-algebra of polynomials in $S$. Let $J(G)$ be the ideal of $P(G)$ generated by all monomials in $S$ and differences of monomials in $S$ of the forms

AMS 1969 subject classifications. Primary 0535.

Key words and phrases. Combinatorial geometry, matroid, geometric lattice, commutative algebra, graded algebra, cohomology.

1 This research was partially supported by NSF Grant GP-11822. 


$$
\begin{array}{ll}
x_{1}^{\nu_{1}} x_{2}^{\nu_{2}} \cdots x_{n}^{\nu_{n}} & \text { where } r\left(V_{x_{i}}\right)<\nu_{1}+\nu_{2}+\cdots+\nu_{n}, \\
x_{1} x_{2} \cdots x_{n}-y_{1} y_{2} \cdots y_{n} & \text { where } V_{x_{i}}=\mathrm{V}_{y_{i}}
\end{array}
$$

respectively and where $n$ is any positive integer. Let $A(G)$ $=P(G) / J(G) . J(G)$ is a homogeneous ideal of the graded algebra $P(G)$, and so $A(G)$ is a graded algebra. For each nonnegative integer $n$, let $A_{n}(G)$ denote the homogeneous component of $A(G)$ of degree $n$. That $A_{n}(G)=0$, for $n>r(G)$, follows from the definition of $J(G)$. The image of $S$ in $A(G)$ generates $A(G)$ as an algebra, and it is easily seen that the natural algebra homomorphism of $P(G)$ onto $A(G)$ embeds $S$ in $A(G)$ as a linearly independent subset. For this reason, identical symbols will be used to denote an element of $S$ and the image of that element in $A(G)$ and $P(G)$.

For any nonnegative integer $n$ such that $n \leqq r(L)$, let $L_{n}$ denote the set of elements of $L$ of rank $n$. For each $x \in L_{n}$, choose $x_{1}, x_{2}, \cdots$, $x_{n} \in S$ such that $x=\vee x_{i}$. The following proposition can easily be proved.

Proposition 1. $\left\{\prod x_{i} \mid x \in L_{n}\right\}$ is a basis for $A_{n}(G)$.

Hence, $A(G)$ has a basis, and $A(G)$ is finite dimensional if and only if $S$ is finite.

For $0 \leqq n \leqq r(L)$, let $B_{n}(G)$ denote the free $k$-module on $L_{n}$. Let $B(G)=\bigoplus B_{n}(G)$. For $x \in L_{n}, y \in L_{m}$ with $n, m \leqq r(L)$, the map

$$
\begin{array}{ll}
(x, y) \mapsto x \vee y, & \text { if } r(x \vee y)=n+m, \\
(x, y) \mapsto 0, & \text { if } r(x \vee y)<n+m,
\end{array}
$$

determines a graded $k$-algebra structure on $B(G)$.

Corollary 1. $A(G) \simeq B(G)$ as graded k-algebras.

Let $\sigma:(G, S) \rightarrow\left(G^{\prime}, S^{\prime}\right)$ be a morphism of geometries. For $x \in S$, the association

$$
\begin{array}{ll}
x \mapsto \sigma(x) \in A\left(G^{\prime}\right), & \text { if } \sigma(x) \in S^{\prime}, \\
x \mapsto 0 \in A\left(G^{\prime}\right), & \text { if } \sigma(x)=0,
\end{array}
$$

uniquely determines an algebra homomorphism of $P(G)$ into $A\left(G^{\prime}\right)$. Since $\sigma$, considered as a strong map of $L(G)$ to $L\left(G^{\prime}\right)$, is supremumpreserving and satisfies $r(\sigma(w)) \leqq r(w)$ for any $w \in L(G)$, the kernel of this algebra homomorphism contains $J(G)$. So there is uniquely determined a homomorphism

$$
A(\sigma): A(G) \rightarrow A\left(G^{\prime}\right)
$$


of algebras. In fact, $A(\sigma)$ is a homomorphism of graded algebras. It follows easily that

PROPOSITION 2. The association $\sigma \mapsto A(\sigma)$ is a functor from the category of geometries, or equivalently the category of geometric lattices and strong maps, to the category of commutative k-algebras.

Proposition 3. If $\left(G \oplus G^{\prime}, S \cup S^{\prime}\right)$ is the coproduct (direct sum) in the category of geometries of $(G, S)$ and $\left(G^{\prime}, S^{\prime}\right)$, then $A\left(G \oplus G^{\prime}\right) \simeq A(G)$ $\otimes A\left(G^{\prime}\right)$. That is, $A$ preserves coproducts.

Proof. $L\left(G \oplus G^{\prime}\right) \simeq L(G) \times L\left(G^{\prime}\right)$ where the lattice structure on the cartesian product is defined componentwise. The linear map of $B\left(G \oplus G^{\prime}\right)$ to $B(G) \otimes B\left(G^{\prime}\right)$ determined by $\left(x, x^{\prime}\right) \mapsto x \otimes x^{\prime}$, where $x \in L(G)$ and $x^{\prime} \in L\left(G^{\prime}\right)$, is easily seen to be an algebra isomorphism, and the proposition follows from Corollary 1.

Notice that $A(G) \otimes A\left(G^{\prime}\right)$ is the tensor product of commutative algebras and is not the tensor product of graded algebras. It is for this reason that $A$ is considered a functor into the category of commutative algebras rather than into the category of graded algebras.

Let $e$ be a closed subset of $S$ where $(G, S)$ is a geometry. So $e \in L(G)$. If $r(e)=n>0$, choose $e_{1}, e_{2}, \cdots, e_{n} \in S$ such that $e=V e_{i}$. Let $\operatorname{ann}(e)$ denote the annihilator of $\prod e_{i}$ in $A(G)$. That is,

$$
\operatorname{ann}(e)=\left\{y \in A(G) \mid y\left(\prod e_{i}\right)=0\right\} \text {. }
$$

$\operatorname{ann}(e)$ is a homogeneous ideal of $A(G)$. Let $\left(G_{e}, e\right)$ denote the subgeometry $[1$, p. 4.1$]$ of $(G, S)$ on the closed subset $e$. Let $A(G)_{e}$ denote the subalgebra of $A(G)$ generated by the subset $e$ of $S$. Let $\left(G / e, S_{e}\right)$ denote the geometry associated to the contraction $[1, \mathrm{p} .4 .1]$ of $(G, S)$ to the open subset $S-e$ (the contraction is, in general, only a pregeometry).

Proposition 4. $A\left(G_{e}\right) \simeq A(G)_{e}$.

Proof. The image of the linear map of $P\left(G_{e}\right)$ into $A(G)$ induced by $\prod x_{i}^{\nu_{i} \mapsto \prod} x_{i}^{\nu_{i}}$, where each $x_{i} \in e$, is $A(G)_{e}$, and its kernel is precisely $J\left(G_{e}\right)$.

Proposition 5. $A(G / e) \simeq A(G) / \operatorname{ann}(e)$.

Proof. The geometric lattice $L(G / e)$ is isomorphic to the interval $[e, 1](1=S)$ of $L(G)[1$, p. 4.2]. The map $\sigma: L(G) \rightarrow[e, 1]$ defined by $\sigma(x)=x \vee e$ is a strong map. The induced surjection $A(\sigma): A(G)$ $\rightarrow A(G / e)$ given by $x \mapsto x\left(\prod e_{i}\right), x \in A(G)$, has kernel ann $(e)$.

Propositions 4 and 5 also hold when the closed subset $e$ is replaced by an arbitrary subset. 
3. Cohomology relative to a point of $(G, S)$. Let $p \in S$ where $(G, S)$ is a geometry. Define $\partial(p) \in \operatorname{End}_{k}(A(G))$ by $\partial(p)(x)=p x, x \in A(G)$. $\partial(p)$ is an endomorphism of degree 1 , and $\partial(p)^{2}=0$ since $p^{2}=0$ in $A(G)$. Letting $m=r(G)$, there is induced a cochain complex

$$
k=A_{0}(G) \stackrel{\partial(p)}{\longrightarrow} A_{1}(G) \stackrel{\partial(p)}{\longrightarrow} \ldots \stackrel{\partial(p)}{\longrightarrow} A_{m-1}(G) \stackrel{\partial(p)}{\longrightarrow} A_{m}(G) .
$$

Let $H^{n}(G, p)=Z^{n}(G, p) / B^{n}(G, p)$ where, for $0<n<m, Z^{n}(G, p)$ is the kernel of $\partial(p): A_{n}(G) \rightarrow A_{n+1}(G)$ and $B^{n}(G, p)$ is the image of $\partial(p): A_{n-1}(G) \rightarrow A_{n}(G)$. Clearly, $Z^{n}(G, p)=\operatorname{ann}_{n}(p)=$ homogeneous component of degree $n$ of $\operatorname{ann}(p)$. Recall that, for $0 \leqq n \leqq m, L_{n}$ denotes the set of elements of $L=L(G)$ of rank $n$ and that, for $x \in L_{n}$, $x_{1}, x_{2}, \cdots, x_{n} \in S$ were chosen so that $x=V_{x_{i}}$. Let $L_{n}(p)$ $=\left\{x \in L_{n} \mid p \leqq x\right\}$. It is easily seen that $\operatorname{ann}_{n}(p), 0<n<m$, has a basis consisting of the set

$$
\left\{\Pi x_{i} \mid x \in L_{n}(p)\right\}
$$

and, for each $w \in L_{n+1}(p)$, the set

$$
\left\{\prod x_{i}-\prod y_{i} \mid y \in L_{n}, x \neq y, y \vee p=w\right\}
$$

where $x \in L_{n}$ is fixed and $x \vee p=w$. Let $|T|$ denote the cardinality of a set $T$. The following proposition is easily proved.

Proposition 6. Let $(G, S)$ be a geometry with $|S|<\infty$. For any $p \in S$ and $0<n<r(G), H^{n}(G, p)$ is a free $k$-module of dimension $\left|L_{n}\right|$ $-\left|L_{n+1}(p)\right|-\left|L_{n}(p)\right|$.

For example, if $(G, S)$ is the free geometry on a finite set $S(L(G)$ = Boolean algebra of all subsets of $S)$, then $H^{n}(G, p)=0$ for all $p \in S$ and $0<n<|S|$.

For a nontrivial example, let $E$ be the set of edges of a finite graph with no loops. Let $(G, E)$ be the bond closure geometry of the graph. That is, if $T \subseteq E$, then the $G$-closure of $T$ consists of all edges both of whose endpoints belong to the same connected component of $T$. Let $e \in E$. Then the dimension of $H^{1}(G, e)$ is the number of 3-circuits (triangles) containing $e$. If the graph is connected and $m$ is the number of edges in a maximal tree ( $m$ is the rank of the geometry), then the dimension of $H^{m-1}(G, e)$ is one less than the number of bonds in the graph containing $e$.

Note also that if $\sigma:(G, S) \rightarrow\left(G^{\prime}, S^{\prime}\right)$ is a morphism of geometries and if $\sigma(p) \neq 0$, then $A(\sigma): A(G) \rightarrow A\left(G^{\prime}\right)$ induces a homomorphism $A(\sigma): H^{n}(G, p) \rightarrow H^{n}\left(G^{\prime}, \sigma(p)\right)$ for each $0<n<r(G)$ where $H^{n}\left(G^{\prime}, \sigma(p)\right)$ $=0$ for $n \geqq r\left(G^{\prime}\right)$. 
4. Remarks. The functor $(G, S) \rightarrow A(G)$ could have been considered as a functor from the category of pregeometries in which case the algebra attached to a pregeometry would be isomorphic to the algebra attached to the associated geometry. The construction of $A(G)$ can be adapted to the definition of geometry in [2] at the expense of clarity of notation.

Can the algebras $A(G)$ be abstractly characterized? Can a characterization be given of the ideals of $A(G)$ arising as kernels of $A(\sigma)$ $: A(G) \rightarrow A\left(G^{\prime}\right)$ where $\sigma:(G, S) \rightarrow\left(G^{\prime}, S^{\prime}\right)$ is a map of geometries? Can $(G, S)$ be recovered from $A(G)$ ? For which $(G, S)$ is $H^{n}(G, p)$ independent of $p \in S$ ?

\section{REFERENCES}

1. H. Crapo and G.-C. Rota, Combinatorial geometries, M.I.T. Press, Cambridge, Mass.

2. W. Graves, A categorical approach to combinatorial geometries, J. Combinatorial Theory (to appear).

University of North Carolina at Chapel Hill, Chapel Hill, North Carolina 27514 